\title{
Performance Analysis of different Variants of SIMPLE Protocol
}

\author{
Neelam Sharma \\ Research Scholar and \\ Corresponding Author, \\ UTU, Dehradun, India
}

\author{
Karan Singh \\ Departments of CS\&E, \\ Jawaharlal Nehru University \\ (JNU), Delhi, India
}

\author{
B. M. Singh \\ Department of CS\&E, \\ College of Engineering \\ Roorkee, India
}

\begin{abstract}
In this paper, A Wireless Body Sensor Network (WBSN) characterizes an independent system that is used for the purpose of monitoring the daily routine activities of an individual. It comprises of smart sensor nodes which do not have any kind of adverse effect on the daily routine activities and are quite effective in the detection of chronic health problems such as diabetes, heart attack, asthma etc. and to caution the person suffering from diseases in the case of an emergency conditions. In this work, performance analysis of different variants of wireless body area network routing protocol named as SIMPLE is done where distance of the sink from various nodes and residual energy of the nodes decides the forwarding nodes to maximize the throughput. In this work, all the sensors on the body will transfer data to sink node and sink node will transmit data to base station or to the server. The simulation results will be evaluated on the grounds of remaining energy, Throughput and number of dead nodes. The obtained results are also compared with recent published protocols and it has been found that in comparison to SIMPLE and iM-SIMPLE, the Enhanced -Simple protocol E2 (nomenclature used in the paper) has throughput higher than the existing ones respectively.
\end{abstract}

\section{Keywords}

Wireless Body Area Network, Wireless Sensor Network, Body Area Networks, Forwarding Function, Radio Model, Residual Energy and Throughput

\section{INTRODUCTION}

A Wireless Body Sensor Network (WBSN) characterizes an independent system that is used for the purpose of monitoring the daily routine activities of an individual [1-3]. It comprises of smart sensor nodes which do not have any kind of adverse effect on the daily routine activities and are quite effective in the detection of chronic health problems such as diabetes, heart attack, asthma etc. and to caution the person suffering from diseases in the case of an emergency conditions.

The above mentioned networks propose encouraging services in many different areas like research, industries, defence, and business [4]. A very nominal measure of power is consumed by the sensor nodes in WBSN. These sensor nodes are very useful and effective in the applications such as military, entertainment, sports training, bomb diffusers, etc. It is now quite easy with the help of WBSNs, even from a remote position, to monitor movements, activities, and important signals of a human body with the help of internet [5].

In this manner, it is quite helpful in saving money. Due to all these facts, the need of these devices is expanding with the time and a number of elements such as fault assurance, reliability, Quality of Service (QoS) and assurance of security required to be fulfilled. WBSNs have not been capable of fulfilling each one of the above discussed specifications because of the limited resources like power of battery memory, changing topology and fluctuating bandwidth.

WBSN nodes have numerous characteristics that turn them perfect for applications in a great number of developing systems. The WBSN technology is a simple and cost effective technology. It is also an energy efficient scheme and it also supports heterogeneity [1-3].

\section{RELATED WORK}

In WBAN, the single hop communication is presented in [5], to deal with the issue of the single hop communication. As far as multi-hop communication is concerned, it is proposed by Sue et.al. in [6]. A Secure Low-Delay Protocol is proposed by Latre et al. for Multi-hop Wireless Body Area Networks. The structure of this routing protocol is like a spanning tree [7]. Parent nodes drain out their energy at a much rapid pace because of the additional traffic load of children nodes.

Quwaider et al. in [8] suggested a routing protocol which endures to variations in network. Store and forward mechanism is used by them to improve the probability of a data packet to achieve effectively to sink node. All sensor nodes are capable to make storage of a data packet. In source to goal course, every node makes storage of the data packet and transmission to following node. With the storage of a data packet and after this retransmission results more consumption of energy and extend end to end delay.

In [9], A Self-Organization Protocol is proposed for Body Area Networks (ANYBODY). The purpose of this protocol is to limit the transmission of sensor nodes directly to sink. It upgrades the effectiveness of network by varying the selection grounds of $\mathrm{CHs}$

Nabi et al. in [10] proposed a protocol which is more or less similar to store and forward system. They incorporate this store and forward plan with Transmit Power Adaption (TPA). In order to have control on the consumption of transmission power, each node is aware of its neighbours. Nodes make the transmission of data with least power and with a steady quality of link.

In [11], Transmission Power Control (TPC) method as proposed by Nabi et al. is applied. At the point when link quality of a node diminished, an Automatic Repeat Request (ARR) is generated and sent back thus it enables the reretransmission of drop packet. Retransmission of lost packet enhances the network throughput with the cost of consumption of energy.

In [12], [13] Tsouri et al. applied creeping waves to relay data packet. This protocol was proposed by them to reduce the energy consumption of nodes at the time of maintaining the reliability on body link. 
In [14], [15] authors make the analysis of the delay in WBANs and various medium access methods for WBAN. A delay tolerant protocol is proposed in [16] by author. They made the comparison of their protocol with various protocols of present.

Later part of this Paper explains the three recently proposed protocols; SIMPLE, IM-SIMPLE AND Enhanced -Simple protocol for WBSN in detail.

\section{SIMPLE PROTOCOL}

In WBANs, the constant quantities of nodes offers chance to loosen up limitations in the case of routing protocols. With the inspiration of routing constrains, SIMPLE protocol enhance the period of stability and throughput of the network. Following paragraphs throws a light on the features of the framework model along with the characteristics of SIMPLE protocol [18].

\subsection{System model}

Fig. 1 illustrates that eight nodes are placed on the body of the person. Each sensor node consists of same power and computation capabilities. The Sink node is placed at chest. ECG and Glucose sensors nodes are Node 1 and Node 2 respectively. Data is transmitted directly to sink by these two nodes.

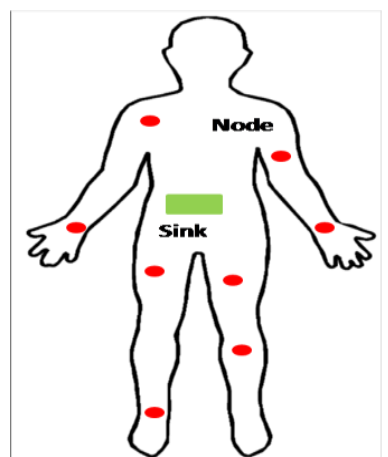

M-ATTEMPT

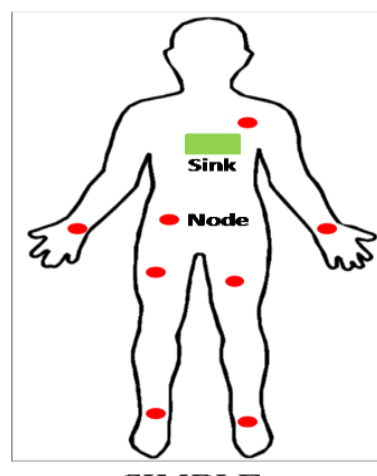

SIMPLE
Fig. 1. Body area network with eight nodes.

The typical sensor node parameters are detailed in Table 1.

Table 1: Radio Parameters [18].

\begin{tabular}{lll}
\hline Parameters & nRF 2401A & CC2420 \\
\hline$E_{T X \text {-elec }}$ & $16.6 \mathrm{~nJ} / \mathrm{bit}$ & $96.89 \mathrm{~nJ} / \mathrm{bit}$ \\
$E_{R X \text {-elec }}$ & $36.2 \mathrm{~nJ} / \mathrm{bit}$ & $172.78 \mathrm{~nJ} / \mathrm{bit}$ \\
$E_{\text {amp }}$ & $1.96 \mathrm{~nJ} / \mathrm{bit}$ & $270.9 \mathrm{~nJ} / \mathrm{bit}$ \\
\hline
\end{tabular}

\subsection{Initial phase}

A small measure of information packet is broadcasted by sink in this phase which comprises the sink's position on the person's body. Each sensor node, after getting the control packet, makes the storage of the sink's position. An information packet is broadcasted by each sensor node which has the following information: position of node on the human body, node ID and the status of the energy. Thus, each sensor node is updated with the information about the location of neighbours and sinks.

\subsection{Selection of next hop}

With the end purpose of saving energy along with the improvement in the network throughput, a multi hop procedure is proposed for WBAN. This section of the paper details about the grounds of selection for a node to turn out to be a parent node or forwarder. The main objective is of creating the balance of the energy consumption among sensor nodes of network. In SIMPLE protocol in each round new forwarder is chosen. Sink node is aware of the information of the nodes such as distance, ID and residual energy status. Sink processes each node's cost function and transmission of this cost function is done to all nodes by sink.

Each node makes the decision on the grounds of the below discussed cost function whether to be a forwarder node or not. Considering ' $i$ ' represents the number of nodes than the evaluation of cost function of node ' $i$ ' is done as follows:

$$
C F(i)=\frac{d(i)}{R E(i)}
$$

In the above equation, the distance between the sink and node ' $i$ ' is represented by $d(i)$ and $R E(i)$ is the residual energy of node ' $i$ ' and is estimated by extracting out the present energy of node from whole energy at the initial phase. We prefer a node with least cost function to be as a forwarder. Each one of the neighbour node get affixed along with the forwarder node and transfer the data possessed by them to forwarder. This data is collected and forwarded to sink by the forwarder. This (forwarder) node contains highest residual energy and least distance to sink; hence, minimum energy is consumed by it in the process of forwarding data to sink. Nodes for Glucose and ECG monitoring establish straightforward communication with sink and do not get indulge in the process of forwarding data.

\subsection{Scheduling}

As far as this phase is concerned, a Time Division Multiple Access (TDMA) is assigned by forwarder node to its children nodes on the basis of the time slots. Each one of these children nodes transmit the data which is sensed by them to forwarder node in its particular predefined time slot. In the case, of the event that a node does not contain any data to be sent, it switches to idle state. Nodes wake up just at the time of its transmission. The dissipation of energy of particular sensor node could be minimized by scheduling of sensor nodes.

\section{IM-SIMPLE PROTOCOL}

In this work, we expand SIMPLE protocol, since SIMPLE protocol does not consider; mobility and appropriate numerical models for energy utilization minimization and in addition throughput expansion

.In IM-SIMPLE PROTOCOL initial phases, estimation of cost function, scheduling and data transmission phase are same.

\subsection{Mobility support}

WBANs are little scale networks in which node move because of the movement of human body which makes the network topology redesign. The mobility pattern of human body is hard to predict. Nodes' positions change as the body moves. To course information effectivelyy, nodes make wireless connection with their closest neighbor

The detected information is lost, when a mobile node neglects to discover its neighbors. To deal with such circumstance, routing protocol must help mobility.

\subsection{Radio Model}

Radio model is used to consider the behaviour of the wireless medium. In past various radio model is proposed for WSN. In 
general first order radio is found suitable for WBAN as detailed in [18-20].

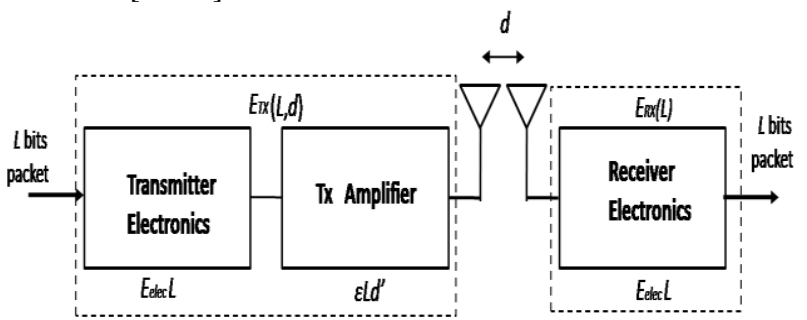

Fig. 2. First order radio model.

The above mentioned radio model assume that $d$, is the separation between transmitter and receiver and, $d^{2}$ represent the loss of energy because of the transmission channel. We can define the first order radio model equations as

$$
\begin{gathered}
E_{T X}(L, d)=E_{T X-\text { elec }}(L)+E_{T X-a m p}(L, d) \\
E_{T X}(L, d)=E_{T X-\text { elec }} \times L+E_{T X-a m p} \times L \times d^{2} \\
E_{R X}(L, d)=E_{R X-\text { elec }}(L) E_{R X}(L)=E_{R X \text {-elec }}(L) \times L
\end{gathered}
$$

Where $E_{T X}$ is the energy consumed at the time of transmission, $E_{R X}$ is the energy consumed during reception, $E_{T X \text {-elec }}$ and $E_{\mathrm{RX} \text {-elec }}$ are the energies needed for the operation of the electronic circuit of transmitter and receiver, respectively. $E_{\text {amp }}$ is termed as the measure of energy needed for the amplifier circuit. On the other side, $L$ denotes the size of the packet.

The platform that is used for the communication in WBAN is human body which provides its contribution of attenuation to radio signal. Hence, path loss coefficient parameter is added by us in radio model. We can rewrite the equation 3 of transmitter as follows

$$
E_{T X}(L, d)=E_{\text {elec }} \times L+E_{\text {amp }} \times L \times d^{n}
$$

The parameters of energy provided in equation 5 rely on the hardware. In WBAN technology, two transceivers that are generally used for the analysis are Nordic nRF 2401A is a single chip, low power and Chipcon CC2420 transceivers. Both have the same bandwidth i.e. $2.4 \mathrm{GHz}$.

Table 2: Simulation parameters.

\begin{tabular}{ll}
\hline Parameter & Value \\
\hline$E_{0}$ & $0.49 \mathrm{Joule}$ \\
$E_{\text {elec }}$ & $5.0 \mathrm{~nJ} / \mathrm{bit}$ \\
$E_{f s}$ & $10.0 \mathrm{pJ} / \mathrm{bit} / \mathrm{m}^{2}$ \\
$E_{a m p}$ & $1.3 \mathrm{fJ} / \mathrm{bit} / \mathrm{m}^{4}$ \\
$E_{d a}$ & $5.0 \mathrm{pJ} / \mathrm{bit}$ \\
Packet Size & $4000 \mathrm{bits}$ \\
\hline
\end{tabular}

\subsection{ENHANCED - SIMPLE PROTOCOL}

The above discussion is based on the protocol forwarding functions. Still a careful analysis is needed which investigates the effect the distance and energy. In order to achieve the target of enhancing the throughput and reliable communication with more reliability between sensors and sink, latest schemes are proposed by us. The various

\begin{tabular}{|c|c|c|}
\hline $\begin{array}{l}\text { Nomenclatur } \\
\text { e }\end{array}$ & Forwarding Function & $\begin{array}{l}\text { Dependenc } \\
\text { y }\end{array}$ \\
\hline CF1 & $F \cdot F(i)=\frac{d(i)}{R \cdot E(i)}$ & $\begin{array}{l}\text { Distance } \\
\text { and } \\
\text { Residual } \\
\text { Energy }\end{array}$ \\
\hline $\mathrm{CF} 2$ & $F \cdot F(i)=\frac{1}{d(i)}$ & $\begin{array}{l}\text { Distance } \\
\text { Only }\end{array}$ \\
\hline CF3 & $F \cdot F(i)=\frac{1}{R \cdot E(i)}$ & $\begin{array}{l}\text { Residual } \\
\text { Energy }\end{array}$ \\
\hline $\mathrm{CF} 4$ & $F . F(i)=\frac{1}{d(i)[R \cdot E(i)]^{2}}$ & $\begin{array}{l}\text { Distance } \\
\text { and } \\
\text { Residual } \\
\text { Energy }\end{array}$ \\
\hline CF5 & $F . F(i)=\frac{1}{d(i)[E-R \cdot E(i)]^{2}}$ & $\begin{array}{l}\text { Distance } \\
\text { and } \\
\text { Dissipated } \\
\text { Energy }\end{array}$ \\
\hline
\end{tabular}
forwarding functions with their nomenclature are detailed in Table 3.
Table 3: Forwarding Functions Comparison.

The CF1 depends on distance and residual energy, CF2 depends on distance only, CF3 depends on residual energy only, CF4 relies on distance and scaled residual energy and CF5 depends on distance and dissipated energy. Form Fig. 3, it is clear that, the performance of CF1 is poorest, and the performance of CF5 is best among the chosen forwarding functions. The performance of $\mathrm{CF} 1$ and $\mathrm{CF} 3$ is nearly same, and on the other side the performance of CF2, CF4 and CF5 is nearly same.

Thus from CF1 and CF3 it can be concluded that, only with residual energy performance is poorer. The performance of CF2 and CF4 is overlapping and not visible in graph. It clearly concluded that the effect so residual energy will have little or no effect. This is very obvious as each of the node have same initial energy, thus their dissipation will also be nearly uniform. In CF5 we try to minimize the dissipated energy, and the performance of the protocol is best among the chosen one.

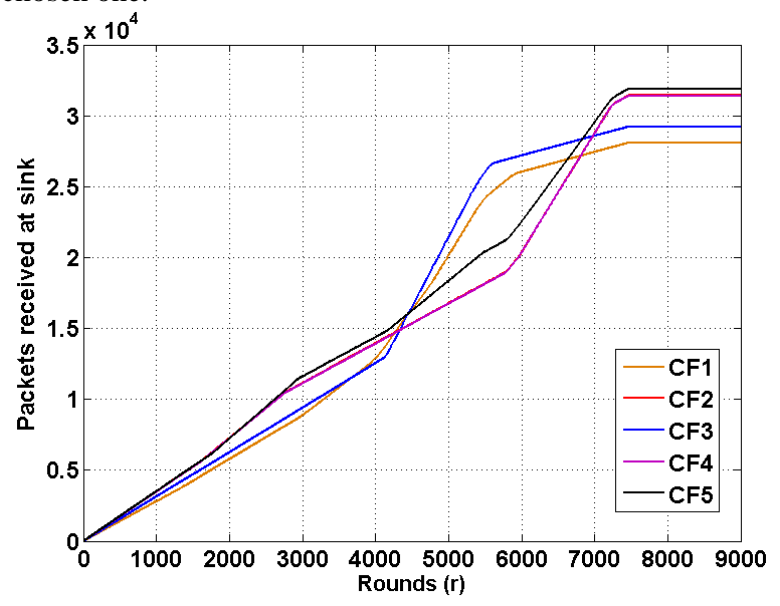

Fig. 3. Throughput comparison of various forwarding functions.

As discussed above, the cost functions CF4 and CF5 have nearly same performance. To generalize the proposed model we modify CF4 and now defined as E1 protocol with forwarding function as:

$F F(i)=\frac{1}{d(i)[R \cdot E(i)]^{p}}, \quad p \geq 2$ 
As it is useless to select a forwarding node which is far away from the data sending node therefore forwarding nodes are selected which are closest to transmitting node.

As a second modification we define forwarding function as

$$
F F(i)=\frac{1}{d(i)[E-R \cdot E(i)]^{p}}, \quad p \geq 2
$$

We defined this protocol as E2. In this function we consider that the energy lost which is defined as difference in the energy of initial energy and left over energy after each round. Also the nodes with lesser energy should not be considered as forwarding node.

\section{SIMULATION RESULTS AND ANALYSIS}

In the simulation various values of index $p$ are considered. However, same results are found for all values greater than or equal to 2, thus we fix it to 2 . The sensor node distribution (network) is shown in Fig.1. Various simulation parameters are detailed in Table 2. The pseudo code for ATTEMPT and SIMPLE and proposed protocol is detailed below:

\begin{tabular}{lc}
\hline $\begin{array}{l}\text { Algorithm } \\
\text { SIMPLE }\end{array}$ & : SIMPLE/EHANCED \\
\hline 1. & Routing Phase \\
2. & In each round \\
3. & For each node \\
4. & Select forwarder using FF \\
5. & if $($ min FF1<minFF2) \\
6. & select node1=forwarder node \\
7. & else \\
8. & select node2=forwarder node \\
9. & if $($ min FF2<minFF1) \\
10. & select node2=forwarder node \\
11. & else \\
12. & select node1=forwarder node \\
13. & $E_{\mathrm{th}} \leftarrow$ Threshold Energy \\
14. & if FF node Energy $>\mathrm{E}_{\mathrm{yh}}$ \\
15. & select node as forwarder \\
16. & else \\
17. & Directly transmit to sink \\
18. & endif \\
19. & endif \\
20. & endif
\end{tabular}

With keeping in mind the end goal of evaluation of the protocols we have carried out an extensive a group of experiments by making use of MATLAB R2010a.

\subsection{Performance Metrics}

The protocol is discussed in terms of the following parameters.

1) Throughput: Throughput is a fractional value and it is the aggregate number of generated packets that are correctly reached at sink. This is also equal to the difference of total generated and loss packets.

2) Residual Energy: Residual energy represents the energy that is left over with the nodes with each subsequent round. This is helpful in evaluating the energy consumption in the network.

\subsection{Enhanced-Simple Protocol In}

\section{Comparison With Simple Protocol}

In this section, results of proposed model are compared with SIMPLE protocol. In Fig. 7, number of dead nodes vs. number of rounds is plotted. In ATTEMPT protocol at the round 2161, all of sudden 3 nodes become dead. However, in case of SIMPLE and E1 till rounds 4436 number of dead node remains as zero. With SIMPLE protocol, the numbers of dead nodes become 6, around the rounds 5917. In E2 till round 2938 number of dead nodes remain zero, and 2939 to 5470 rounds the number of dead node is one only. From round 5471 to 7222 numbers of dead nodes are two. There after it catches other protocols.

In Fig. 8, residual energy vs. round is plotted. Here the residual energy curve for SIMPLE and E1 shows similar behaviour and finally residual energy goes to zero at 7445 rounds. Thus in terms of residual energy saving as such no advantage gain with E1. However the E2 protocol has better saving of energy due to lesser number of dead nodes.

In Fig. 9 packet received at the sink vs. Round is plotted, it is clear from the figure that the performance of the proposed protocols is better in comparison to the SIMPLE protocol. After 6000 rounds a significant difference in the packet received at the sink can be observed. In case of E1 protocol the total packets received are $3.06 \times 10^{4}$, while for SIMPLE protocol; the numbers of received packets are $2.8 \times 10^{4}$. Therefore, a rise in the packets received and in terms of percentage it is $9.28 \%$. However, in case of E2, the numbers of received packets are $3.21 \times 10^{4}$ which is 12.46 better than SIMPLE protocol.

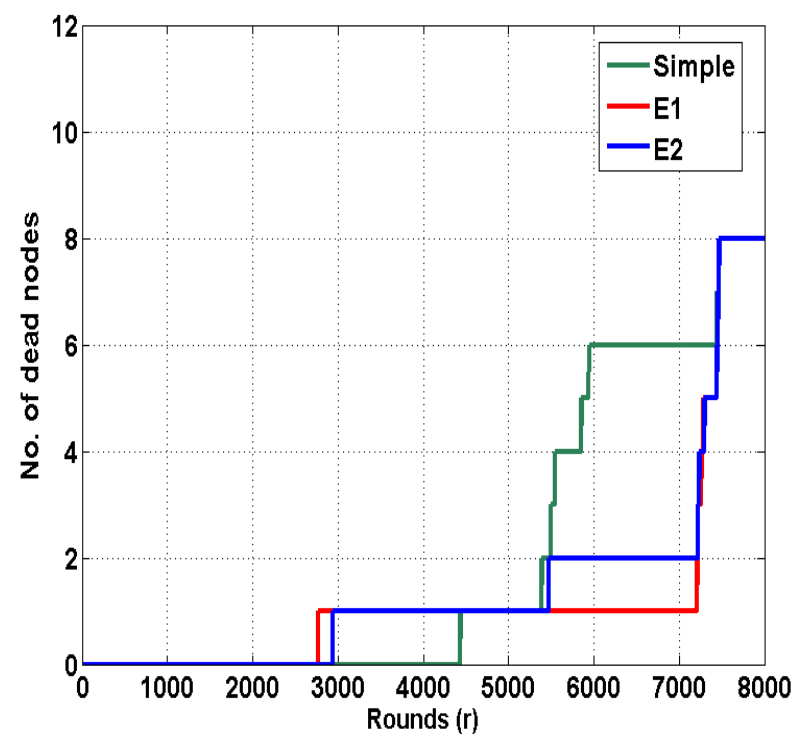

Fig. 7. No. of dead nodes vs. rounds 


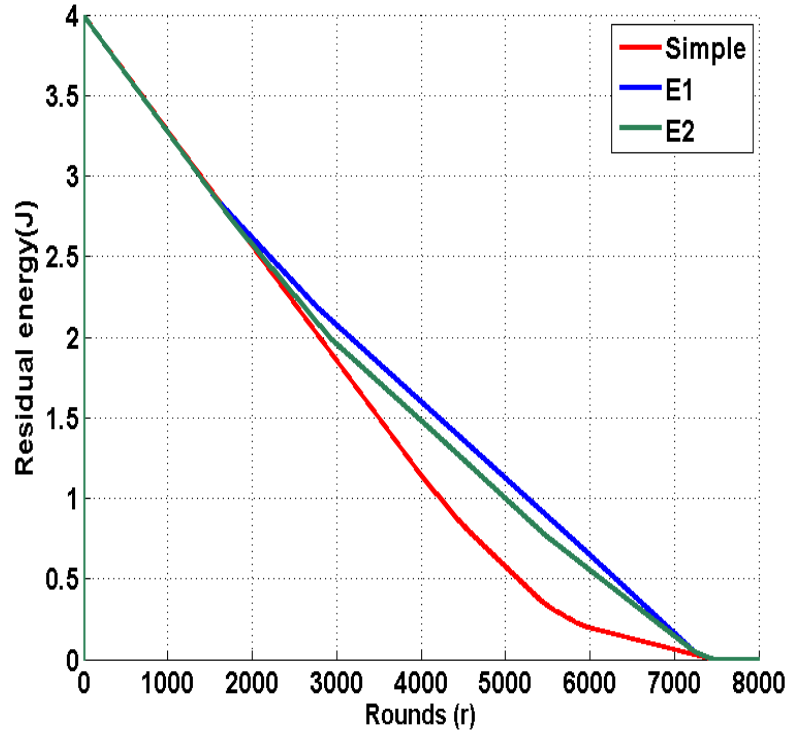

Fig. 8. Residual energy vs. rounds

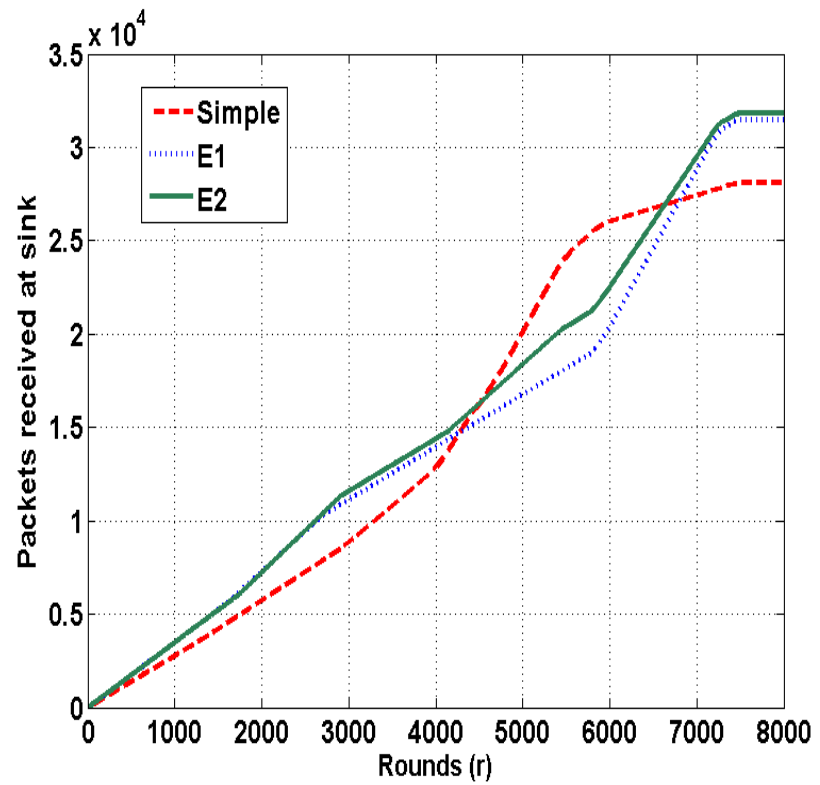

Fig. 9. Packet received at the sink vs. rounds

\section{COMPARISON WITH RECENT PROTOCOLS}

The comparison of recently published protocols is shown in Fig. 10. Very recently in iM-SIMPLE protocol is proposed where maximum possible throughput is $3.0 \times 10^{4}$ [21].

Thus, still our protocols E1-performance is $2 \%$ better than the iM-SIMPLE, and E2 performance is $6.7 \%$ better than the iMSIMPLE. The performance of E2-SIMPLE is $12.46 \%$ better than SIMPLE protocols.

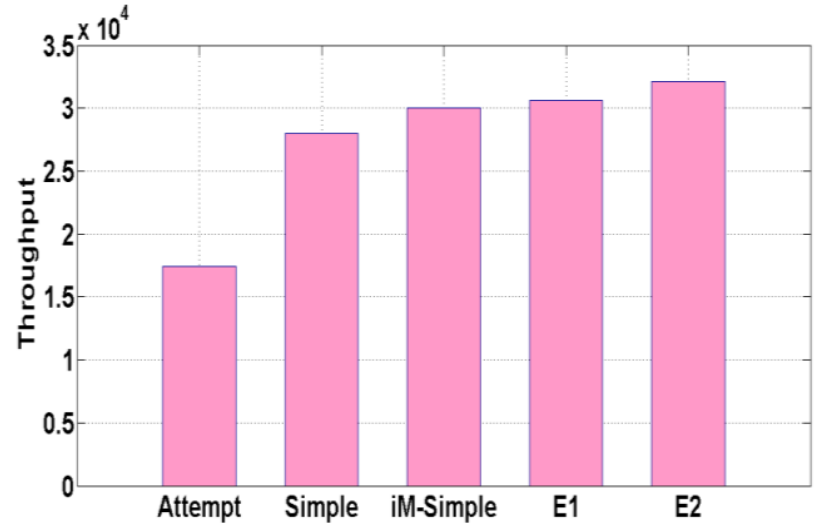

Fig. 10. Throughput comparison of recent protocols.

\section{CONCLUSION}

In this work an energy efficient wireless body area network protocol is presented. The selection of forwarding nodes is based on the cost function which is dependent on the distance and energy. The major conclusions can be summarized as follows:

- It is found that the proposed protocol works well in comparison to earlier proposed protocol SIMPLE and iM-SIMPLE.

- The throughput of the proposed protocol is nearly $12.46 \%$ more than the SIMPLE protocol. However, in comparison to iM-SIMPLE the E2-SIMPLE has $6.7 \%$ better throughput.

- In the major conclusions, it is also found that the residual energy have no or little effect on throughput. However, distance has significant effect on the throughput.

\section{REFERENCES}

[1] Ghamari, M.; Janko, B.; Sherratt, R.S.; Harwin, W.; Piechockic, R.; and Soltanpur, C. (2016). A survey on wireless body area networks for ehealthcare systems in residential environments. Sensors, 16, 831.

[2] Salehi, S. A.; MRazzaque, A.; Tomeo-Reyes, I.; and Hussain, N. (2016). IEEE 802.15.6 standard in wireless body area networks from a healthcare point of view. 22nd Asia-Pacific Conference on Communications (APCC), Yogyakarta, 523-528.

[3] Alam, M. M.; and Hamida, E. B. (2014). Surveying wearable human assistive technology for life and safety critical applications: Standards, challenges and opportunities. Sensors, 14(5), 9153-9209.

[4] Adibi, S. (Ed.). (2015). Mobile Health: A Technology Road Map. 5, Springer.

[5] Quwaider, M.; and Biswas, S. (2010). DTN routing in body sensor networks with dynamic postural partitioning. Ad Hoc Networks, 8(8), 824841.

[6] Seo, S. H.; Gopalan, S.; Chun, S. M.; Seok, K. J.; Nah, J. W.; Park, J. T. (2010). An energy-efficient configuration management for multihop wireless body area networks. Broadband Network and Multimedia Technology (IC-BNMT), 3rd IEEE International Conference, 235.

[7] Latre, B.; Moerman, I.; Blondia, C.; Reusens, E.; Joseph, W.; and Demeester, P. (2007). A low-delay protocol for multi-hop wireless body area networks. 
Fourth Annual International Conference on Mobile and Ubiquitous Systems: Networking and Services, 1-8.

[8] Quwaider, M.: and Biswas, S. (2009). On-body packet routing algorithms for body sensor networks. Networks and Communications, IEEE-NETCOM, 171-177.

[9] Watteyne, T.; Augé-Blum, I.; Dohler, M. and Barthel, D. (2007). Anybody: a self-organization protocol for body area networks. Proceedings of the ICST 2nd international conference on Body area networks. ICST (Institute for Computer Sciences, Social-Informatics and Telecommunications Engineering), 6 .

[10] Nabi, M.; Basten, T.; Geilen, M.; Blagojevic, M. and Hendriks, T. (2010). A robust protocol stack for multihop wireless body area networks with transmit power adaptation. Proceedings of the Fifth International Conference on Body Area Networks, 77-83.

[11] Guo, C.; Prasad, R. V. and Jacobsson, M. (2010). Packet forwarding with minimum energy consumption in body area sensor networks. 7th IEEE Consumer Communications and Networking Conference (CCNC), $1-6$.

[12] Tsouri, G.R.; Sapio, A. and Wilczewski, J. (2011). An investigation into relaying of creeping waves for reliable low-power body sensor networking. IEEE Transactions on Biomedical Circuits and Systems, 5(4), 307-319.

[13] Sapio, A.; and Tsouri, G.R. (2010). Low-power body sensor network for wireless ecg based on relaying of creeping waves at 2.4 ghz. 2010 IEEE International Conference on Body Sensor Networks (BSN), 167-173.

[14] Javaid, N.; Yaqoob, M.; Khan, M. Y.; Khan, M. A.; Javaid, A. and Khan, Z. A. (2013). Analyzing delay in wireless multi-hop heterogeneous body area networks. Research Journal of Applied Sciences, Engineering and Technology, arXiv preprint arXiv:1304,1059.

[15] N. Javaid, I. Israr, M. A. Khan, A. Javaid, S. H. Bouk, Z. A. Khan, (2013). Analyzing Medium Access Techniques in Wireless Body Area Networks. Research Journal of Applied Sciences, Engineering and Technology, arXiv preprint arXiv:1304,1059.

[16] Muhannad, Q. and Biswas, S. (2009). Probabilistic routing in on body sensor networks with postural disconnections. Proceedings of the 7th ACM international symposium on Mobility management and wireless access, 149-158.

[17] Javaid, N.; Abbas, Z.; Farid, M.S.; Khan, Z.A.; and Alrajeh, N. (2013). M-ATTEMPT: a new energyefficient routing protocol for wireless body area sensor networks. 4th International Conference on Ambient Systems, Networks and Technologies, Halifax, Nova Scotia, Canada, Procedia Computer Science, 19, 224231.

[18] Nadeem, Q.; and Javaid, N. (2013). SIMPLE: stable increased-throughput multi-hop protocol for link efficiency in broadband and wireless computing. Eighth International Conference on Communication and Applications , 221 - 226. Compiegne, France.

[19] Mainwaring, A. (2002). Wireless sensor networks for habitat monitoring, Proceedings of the 1st ACM international workshop on Wireless sensor networks and applications, 88-97. ACM Press.

[20] Manzoor, B.; Javaid, N.; Rehman, O.; Akbar, M.; Nadeem, Q.; Iqbal, A.; and Ishfaq, M. (2013). QLEACH: a new routing protocol for WSNs. International Workshop on Body Area Sensor Networks in conjunction with 4th International Conference on Ambient Systems, Networks and Technologies. Halifax, Nova Scotia, Canada, Procedia Computer Science, 19, 926-931.

[21] Javaid, N.; Ahmad, A.; Nadeem, Q.; Muhammad, I.; and Noman, H. (2015). iM-SIMPLE: iMproved stable increased-throughput multi-hop link efficient routing protocol for Wireless Body Area Networks, Computers in Human Behavior.51,1003-1011. 